Physics Vol. 2, No. 6, pp. 247-261, 1966. Physics Publishing Co. Printed in Great Britain.

\title{
TUNNELING IN SUPERIMPOSED FILMS
}

\author{
J. J. HAUSER \\ Bell Telephone Laboratories, Incorporated \\ Murray Hill, New Jersey \\ (Received 8 February 1966 )
}

\begin{abstract}
Tunneling experiments were performed on superimposed films of $P b-A l, P b-C d$ and $\mathrm{Pb}-\mathrm{Pt}$ deposited and kept at $77^{\circ} \mathrm{K}$ until measured. The gap in the density of states induced by a $900 \AA$ A thick lead film in the Al, Cd and Pt films was measured as a function of their thickness. The experiments are in fairly good agreement with McMillan's theory, which was derived for pure films and scattering at the normalsuperconducting interface. On the other hand, gapless superconductivity has been observed in lead films in contact with nickel and platinum films. The degree of gapless superconductivity (i.e. the density of states in the lead gap) has been measured from $d V / d I$ versus $V$ curves as a function of the lead film thickness. As expected, a magnetic metal such as nickel has a larger effect on the density of states than a normal metal such as platinum, even if the effect is measured in sandwiches with the same transition temperature. The degree of gapless superconductivity in any of the sandwiches can be increased by applying a magnetic field; the relative conductance $\rho_{s} / \rho_{n}$ (which is a neasure of the degree of gapless superconductivity) increases linearly with magnetic field close to the upper critical field as predicted theoretically by Maki.
\end{abstract}

\section{Introduction}

THE superconducting proximity effect, i.e. the mutual effect of two films on each other has been studied in two different ways. In the first approach, the depression of the transition temperature of a superconducting film by a normal film was measured resistively and such measurements have been used to estimate the elect::on-electron interaction in the normal metal [1-10]. The second approach consists in inducing superconductivity in an otherwise normal metal by an adjoining superconducting film. Such an eff əct was first studied by Meissner [11] who found that contacts between superconductors with a normal film between became superconducting when the thickness of the normal film was very small. Such effects might, however, be attributed to a superconducting bridge occurring across the normal film because of the pressure exerted in making the contact. Smith et al. [12] found superconductivity induced in a silver film by a lead film, both by persistent current measurements and tunneling experiments. Rowell [13] has made tunneling experiments on lead-silver sandwiches evaporated at room temperature and his results have been interpreted in terms of a theory develoyed by McMillan [13]. As previously pointed out [9], experiments on sandwiches evaporated at room temperature may be obscured by various unwanted metallurgical and chemical reactions. It is for this reason that all the sandwiches investigated here were made and kept at $77^{\circ} \mathrm{K}$ until neasured. From the theoretical point of view, 
two quantitative theories have been proposed to discuss induced superconductivity in a normal metal: McMillan's theory [13] for pure films with scattering at the N-S interface and SaintJames' theory [14] for films with specular reflexion at the boundary. It would be interesting, therefore, to study the gap as a function of the normal film thickness and compare the results with existing theories. Furthermore, one may also expect the induced gap to be related in some fashion to the gap the "normal" metal has without the superimposed lead film (by "normal" metal may be meant either a metal in which superconductivity has not been found, e.g. platinum, or a superconductor above its transition temperature, e.g. aluminum and cadmium). One also wonders whether the combination of such measurements with the proper theory would permit a better estimate of the electron-electron interaction in normal metals than the one obtained by the other approach to the proximity effect $[7,8]$.

On the other hand, Woolf and Reif [15] have reported gapless superconductivity and smeared density of states in superimposed films of $\mathrm{Fe}$ and $\mathrm{In}$ and $\mathrm{Mn}$ and $\mathrm{Sn}$. The gapless superconductivity produced by the proximity effect of magnetic films as well as nonmagnetic films was discussed theoretically by Fulde and Maki [16] and de Gennes and Mauro [17]. A quantitative study of the degree of gapless superconductivity (i.e. of the density of states present in the gap) as a function of superconducting film thickness will be reported in this paper and the experimental results compared with available theories $[13,16,17]$.

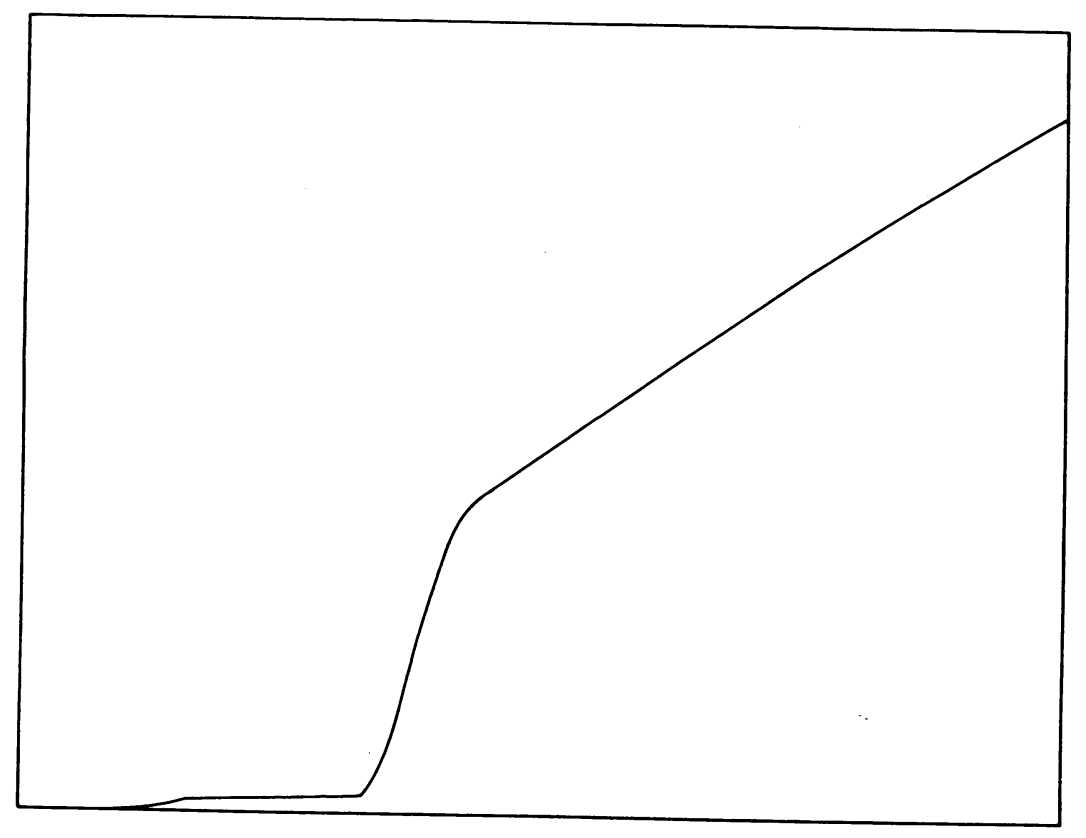

FIGURE 1

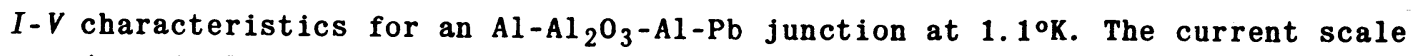
(vertical) is $10 \mu \mathrm{A}$ per large division and the voltage scale is $0.2 \mathrm{mV}$ per large division.

$$
d_{\mathrm{Al}}=35 \stackrel{\circ}{\mathrm{A}} ; \quad d_{\mathrm{Pb}}=900 \stackrel{\circ}{\mathrm{A}} .
$$




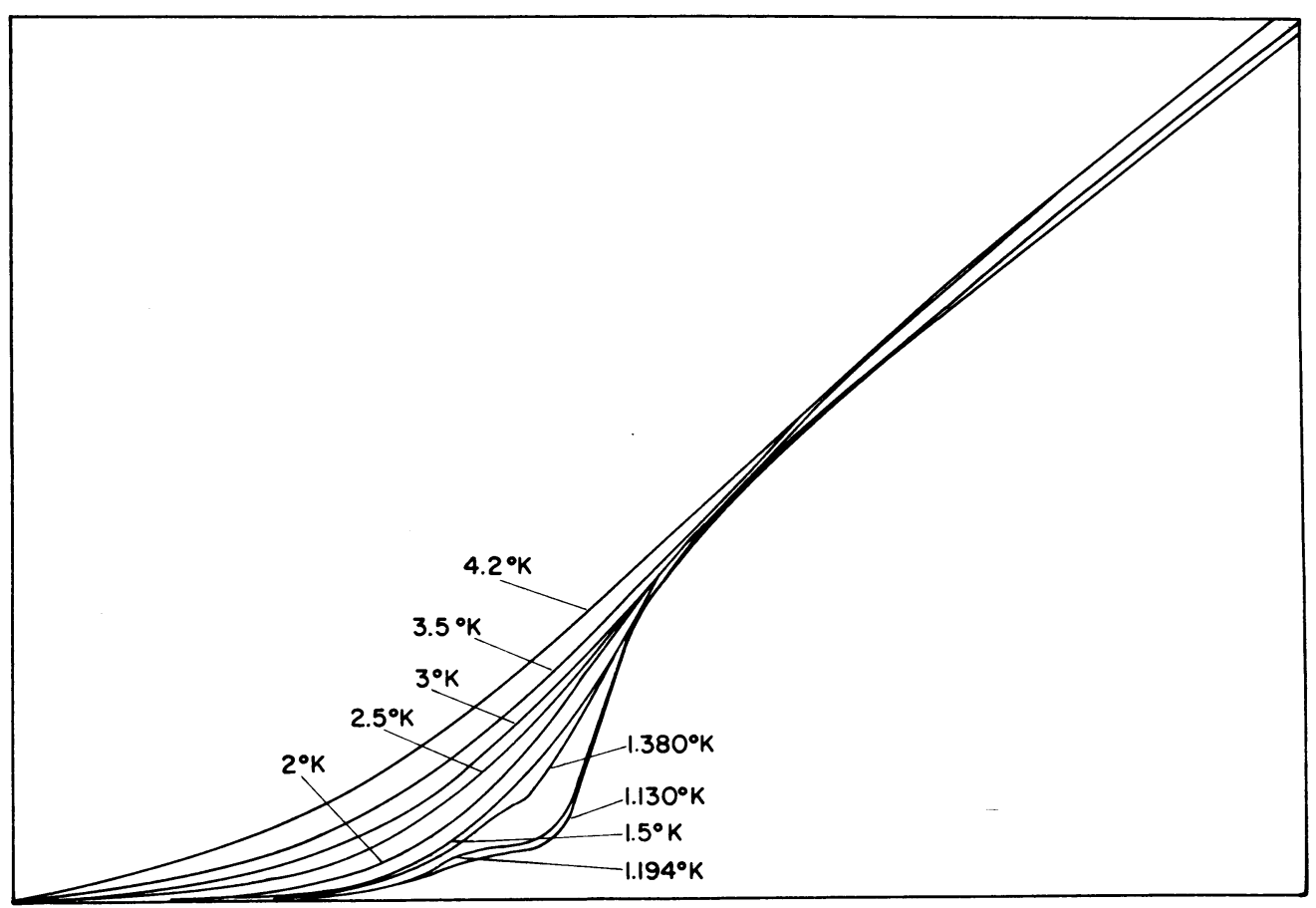

FIGURE 2

I-V characteristics for an $\mathrm{Al}-\mathrm{Al}_{2} \mathrm{O}_{3}-\mathrm{Cd}-\mathrm{Pb}$ junction as a function of temperature. The current scale is $20 \mu \mathrm{A}$ per large division and the voltage scale is $0.2 \mathrm{mV}$ per large division.

$$
d_{C d}=56 \stackrel{\circ}{\mathrm{A} ;} d_{\mathrm{Pb}}=900 \stackrel{\circ}{\mathrm{A}} \text {. }
$$

\section{Experimental Procedure}

In order to produce reproducible low resistance tunnel junctions, the aluminum films used in this investigation were evaporated from a tungsten filament inside a small stainless steel can in a vacuum of $3 \times 10^{-7} \mathrm{~mm}$. The aluminum films were then oxidized by heating at $150^{\circ} \mathrm{C}$ for half an hour in a constant moisture atmosphere. When the sandwich or film was deposited at $77^{\circ} \mathrm{K}$ on such an oxidized aluminum film, the resistance of the junction varied from 30 to $2 \Omega$ depending on the type of metal deposited on the aluminum oxide. The substrates used in this study were glass wafers $1 \times 0.5 \mathrm{~cm}$ and a $1000 \AA$ aluminum film was evaporated as a $0.2 \mathrm{~mm}$ wide diagonal strip. The sandwiches were sputtered at $77^{\circ} \mathrm{K}$ as a $0.3 \mathrm{~mm}$ wide strip along the other diagonal using the getter-sputtering apparatus previously described [9]. The junctions thus prepared were transferred under liquid nitrogen onto a holder with four spring loaded contacts. This permitted the tunneling experiments to be made without ever warming the sandwiches above $77^{\circ} \mathrm{K}$. On the other hand, the sandwiches composing the tunnel junction could also be measured resistively in order to determine their transition temperatures and resistivities. Each film .thickness is determined $[7,9]$ to within $\pm 10 \%$ by timing the duration of deposition and then calibrating the deposition by determining the thickness of a thick film by interferometry. The 
$d V / d I$ versus $V$ curve were obtained using a standard modulation technique with an a.c. sensing signal of about $20 \mu \mathrm{V}$ at a frequency of 10,000 cycles.

\section{Experimental Results}

\section{(a) Gaps induced in otherwise "normal" metals by a superconducting lead film}

In this study we report gaps in the density of states of three metals: Pt, Cd and $\mathrm{Al}$. In all three cases, the tunneling curves were studied on the normal metal side and the overlying lead film was $900 \AA$ thick. The evaporated aluminum film had a transition temperature of $1.4^{\circ} \mathrm{K}$ and a

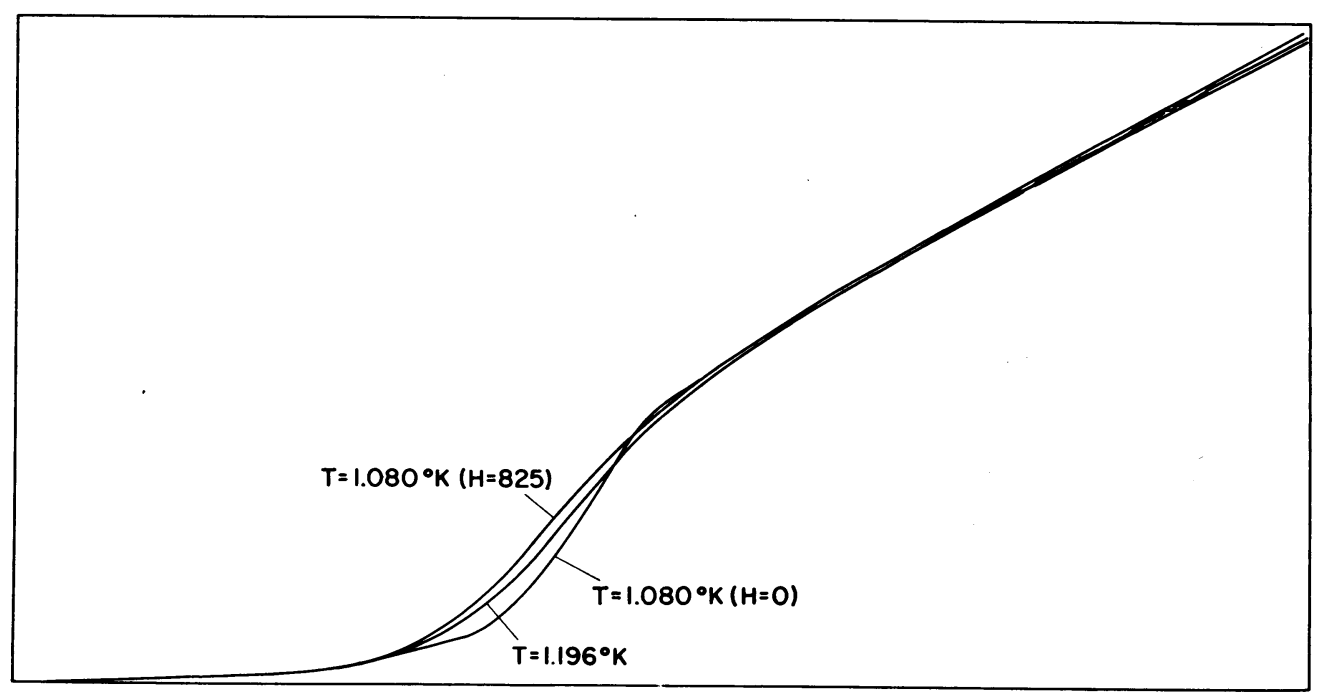

FIGURE 3

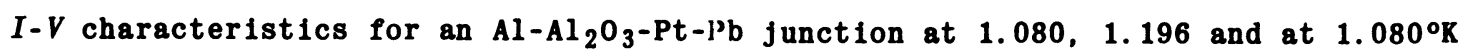
with a parallel magnetic field of 825 0e. The current scale is $0.2 \mathrm{~mA}$ per large division and the voltage scale is $0.2 \mathrm{mV}$ per large division.

$$
d_{\mathrm{Pt}}=14 \stackrel{\circ}{\AA} ; \quad d_{\mathrm{Pb}}=900 \stackrel{\circ}{\mathrm{A}} \text {. }
$$

gap $2 \Delta=0.3 \mathrm{mV}$ at $1.1^{\circ} \mathrm{K}$. The aluminum films composing the Al-Pb sandwiches were gettersputtered at $77^{\circ} \mathrm{K}$; and as a result, their transition temperatures ranged between 2 and $3^{\circ} \mathrm{K}$ with a gap $2 \Delta \simeq 0.66 \mathrm{mV}$ at $1.1^{\circ} \mathrm{K}$. Transition temperatures as high as $3^{\circ} \mathrm{K}$ in aluminum films evaporated at low temperatures have already been reported previously [18]. A typical $I-V$ curve taken on a $\mathrm{Pb}-\mathrm{Al}$ sandwich is shown in Fig. 1. The gap induced by the lead film in the $35 \AA$ aluminum film is approximately $2 \Delta=2.4 \mathrm{mV}(\Delta=1.2 \mathrm{mV}$ being the middle of the almost horizontal portion of the $I-V$ curve between 1.0 and $1.4 \mathrm{mV}$ ). The bump in the $I-V$ curve around $1.6 \mathrm{mV}$ is believed to arise because of the peak in the density of states at the edge of the lead gap. In all the tunneling experiments on superimposed films made on the normal side, one always notices a kink or bump in the $I-V$ curve around $1.5 \pm 0.1 \mathrm{mV}$ which is equal to $\Delta_{\mathrm{pb}}$. The kink or bump is, of 
TABLE 1

Gaps induced in $\mathrm{Al}, \mathrm{Cd}$ and $\mathrm{Pt}$ by a $900 \stackrel{\circ}{\mathrm{Ab}} \mathrm{film}$

\begin{tabular}{|c|c|c|}
\hline$d_{\mathrm{Al}} \stackrel{\circ}{\AA}$ & $\Delta_{\mathrm{Al}}\left(1.1^{\circ} \mathrm{K}\right) \mathrm{mV}$ & $\xi_{A 1} / d$ \\
\hline 35 & 1.2 & 1.05 \\
\hline 70 & 0.85 & 0.62 \\
\hline 140 & 0.67 & 0.35 \\
\hline 280 & 0.55 & 0.19 \\
\hline$\infty$ & 0.32 & 0 \\
\hline$d_{\mathrm{Cd}} \stackrel{\circ}{\AA}$ & $\Delta_{\mathrm{Cd}}\left(1.1^{\circ} \mathrm{K}\right) \mathrm{mV}$ & $\xi_{C d} / d$ \\
\hline 14 & 1.35 & 4.3 \\
\hline 28 & 1.25 & 2.24 \\
\hline 56 & 1.10 & 1.20 \\
\hline 112 & 0.95 & 0.64 \\
\hline 336 & 0.55 & 0.28 \\
\hline 504 & 0.50 & 0.20 \\
\hline$d_{\mathrm{Pt}} \stackrel{\circ}{\mathrm{A}}$ & $\Delta_{\mathrm{Pt}_{\mathrm{t}}}\left(1.1^{\circ} \mathrm{K}\right) \mathrm{mV}$ & $\xi_{\mathrm{Pt}_{\mathrm{t}}} / d$ \\
\hline 14 & 0.925 & 3.5 \\
\hline 35 & 0.54 & 1.83 \\
\hline 70 & 0.315 & 1. 20 \\
\hline
\end{tabular}

course, more pronounced the thinner the normal metal. The summary of the results for $\mathrm{Pb}-\mathrm{Al}$ sandwiches is presented in Table 1. The $\mathrm{Pb}-\mathrm{Al}$ sandwich with $140 \mathrm{~A}$ of aluminum was warmed to room temperature, and the overlying lead film stripped with acetic acid. The junction was then remeasured and the gap in the sputtered aluminum had been reduced to $\Delta_{\mathrm{Al}}\left(1.1^{\circ} \mathrm{K}\right)=0.22 \mathrm{mV}$. This value of the gap is smaller than that of an aluminum film made and kept at $77^{\circ} \mathrm{K}\left(\Delta_{\mathrm{Al}}=0.33 \mathrm{mV}\right)$; this is consistent with the fact that the transition temperature of the aluminum film was also reduced to about $1.5^{\circ} \mathrm{K}$ by warming up to room temperature [18]. Such an experiment clearly demonstrates that the induced gaps are not the result of some diffusion effect. 
A typical example of $I-V$ characteristics for the $\mathrm{Pb}-\mathrm{Cd}$ system is shown in Fig. 2. The gap in the $56 \AA \mathrm{Ad}$ film is $2 \Delta=2.2 \mathrm{mV}$ and one notices again the pronounced kink in the $I-V$ curve at $1.5 \mathrm{mV}$ which becomes more evident the lower the temperature of measurement. Finally, an example of tunneling in a $\mathrm{Pb}-\mathrm{Pt}$ sandwich is shown in Fig. 3; and the results for all Cd and Pt films measured are summarized in Table 1.

\section{(b) Gapless superconductivity in lead films}

In these experiments, the tunneling characteristics are always obtained on the lead side of the sandwich. The normal metals, in the present study platinum and nickel, are kept at a constant thickness of, respectively, 200 and $45 \AA$. The degree of gapless superconductivity can be

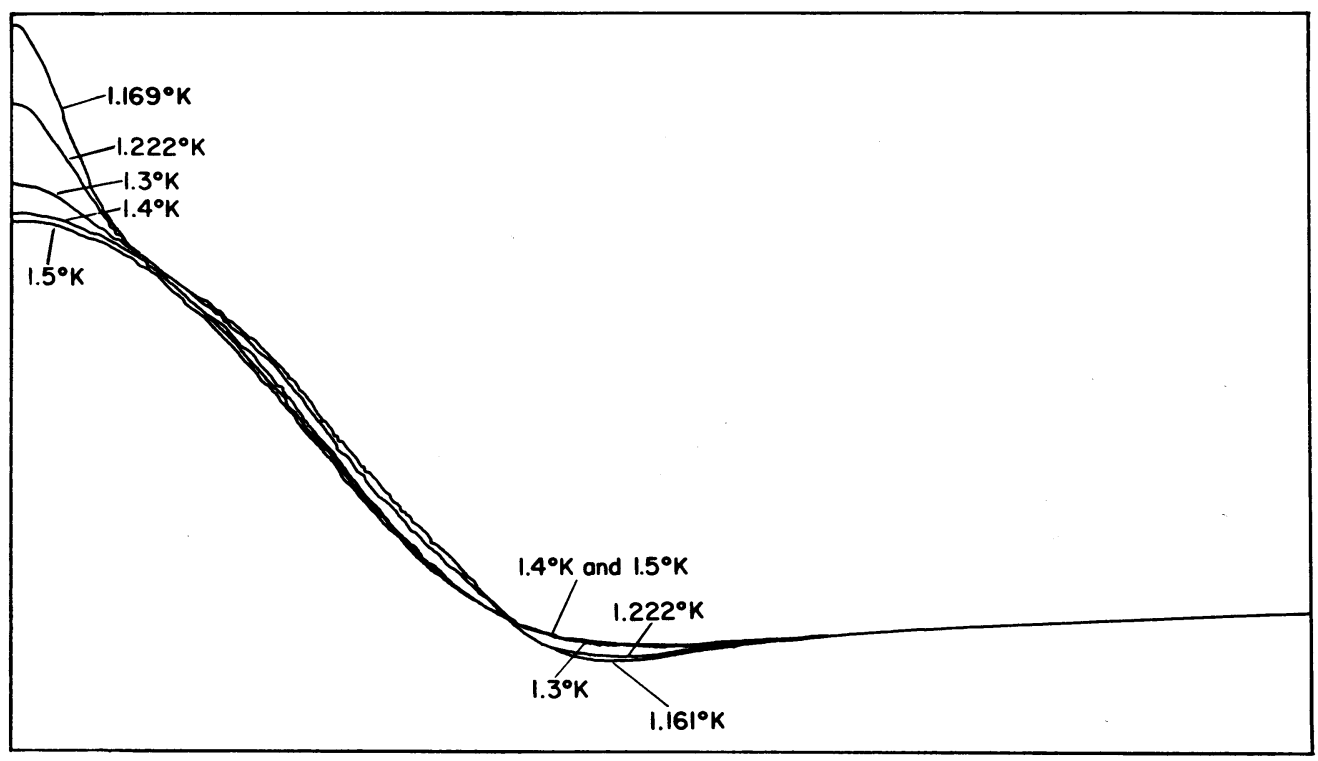

FIGURE 4

$d V / d I-V$ curve for an $\mathrm{Al}-\mathrm{Al}_{2} \mathrm{O}_{3}-\mathrm{Pb}-\mathrm{Ni}$ junction as a function of temperature. The $d V / d I$ scale (vertical) is $2 \mu V$ per large devision at constant current and the voltage scale is $0.2 \mathrm{mV}$ per large division.

$$
d_{\mathrm{Pb}}=600 \stackrel{\circ}{\mathrm{A} ;} \quad d_{\mathrm{Ni}}=45 \stackrel{\circ}{\AA} .
$$

varied by changing the lead film thickness. An example of such gapless superconductivity is shown in Fig. 4 for a $\mathrm{Pb}-\mathrm{Ni}$ sandwich with a lead thickness of $600 \AA$. The ordinate is inversely proportional to the conductance $\rho$, and consequently the relative conductance $\rho_{s} / \rho_{n}$ is obtained by taking the ratio of the ordinate at $3 \mathrm{mV}$ to the ordinate at zero bias. One notices in Fig. 4 that the curves assume a different shape, especially below $0.3 \mathrm{mV}$ bias, when the temperature is lowered below $1.4^{\circ} \mathrm{K}$ (the transition temperature of the measuring aluminum film). Another example of gapless superconductivity in a $\mathrm{Pb}-\mathrm{Ni}$ sandwich with a $1500 \AA$ lead film at $1.1^{\circ} \mathrm{K}$ is shown in Fig. 5. In zero field, when the aluminum is superconducting, one observes around 1.2 $\mathrm{mV}$ a peak which is associated with the peak of the density of states at the edge of the lead gap. This peak disappears completely when the parallel magnetic field is increased to $1.8 \mathrm{kG}$ 
(approximately the critical field of the measuring aluminum film at this temperature); at this point the shape of the curve and the ratio $\rho_{s} / \rho_{n}$ are approximately the same as those one would obtain in zero field at $1.4^{\circ} \mathrm{K}$ as in Fig. 4.

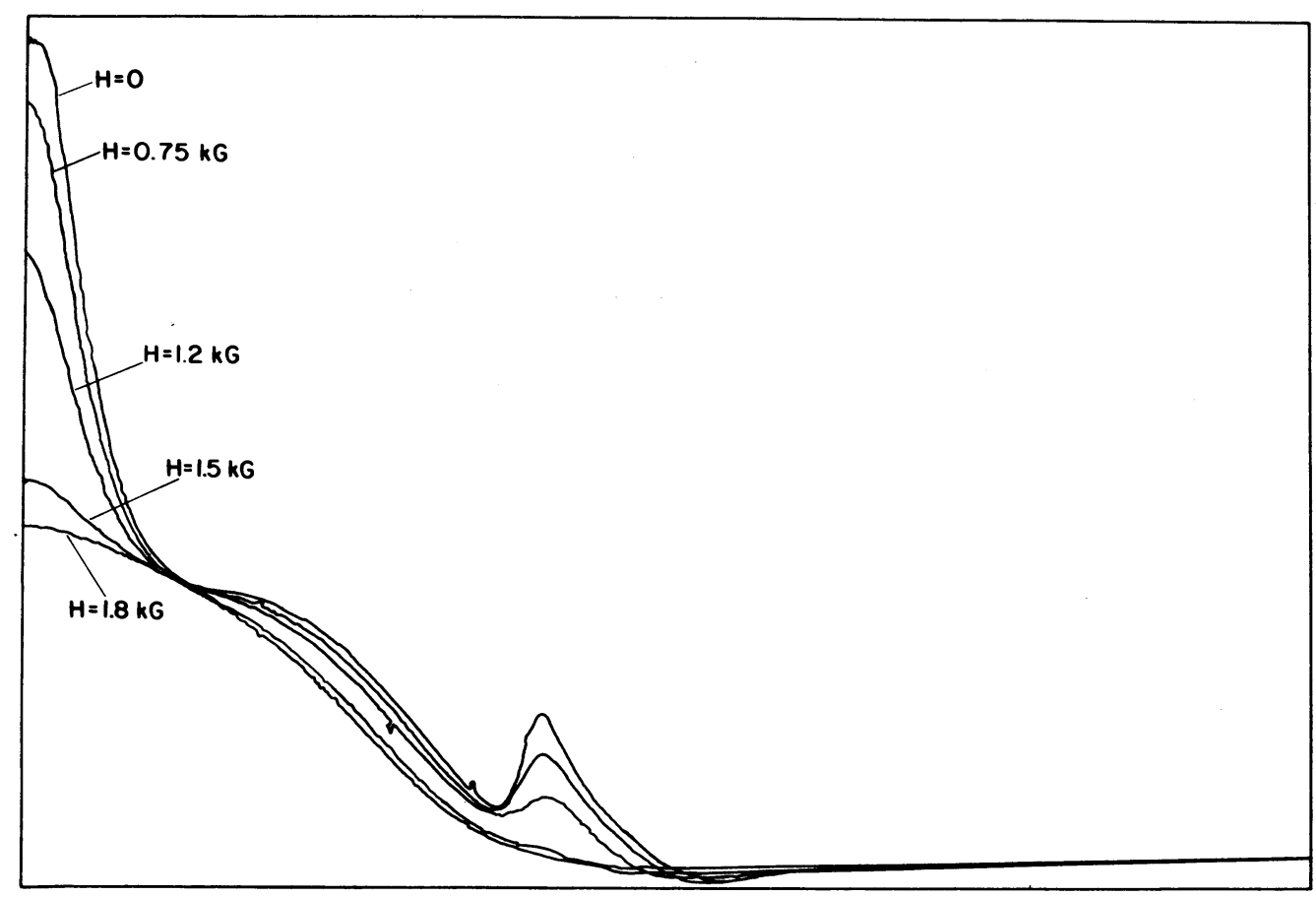

FIGURE 5

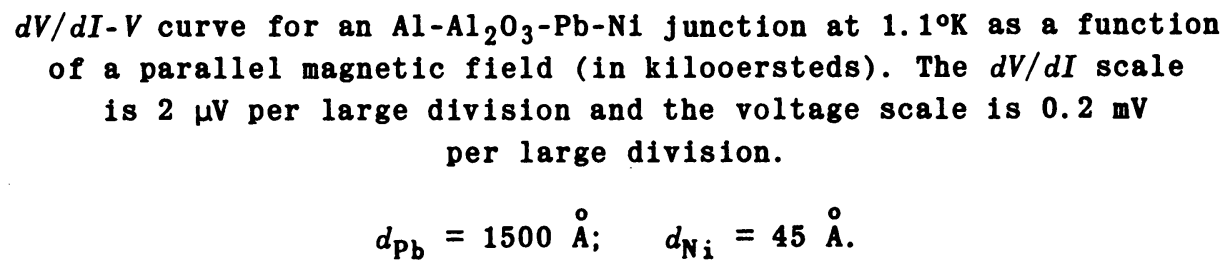

Gapless superconductivity can also arise when a lead film is in contact with a nonmagnetic metal such as platinum as shown in Fig. 6. In magnetic fields below $3 \mathrm{kG}$, one observes a relatively flat peak around $1.2 \mathrm{mV}$ which corresponds to the peak of the density of states at the edge of the lead gap. The degree of gapless superconductivity, as measured by $p_{s} / p_{n}$, is shown as a function of lead film thickness for both $\mathrm{Pb}-\mathrm{Ni}$ and $\mathrm{Pb}-\mathrm{Pt}$ sandwiches in Fig. 7. The manner in which $p_{s} / p_{n}$ was determined for a given sandwich will be discussed later.

Finally, it has also been predicted [19-21] that gapless superconductivity should occur in thin "dirty" films under high magnetic fields. Such an effect has already been reported for thick films or bulk type II superconductors $[22,23]$. The degree of gapless superconductivity as measured by $\rho_{s} / \rho_{n}$ is shown as a function of magnetic field for $\mathrm{Pb}-\mathrm{Ni}$ and $\mathrm{Pb}-\mathrm{Pt}$ sandwiches in Fig. 8. 


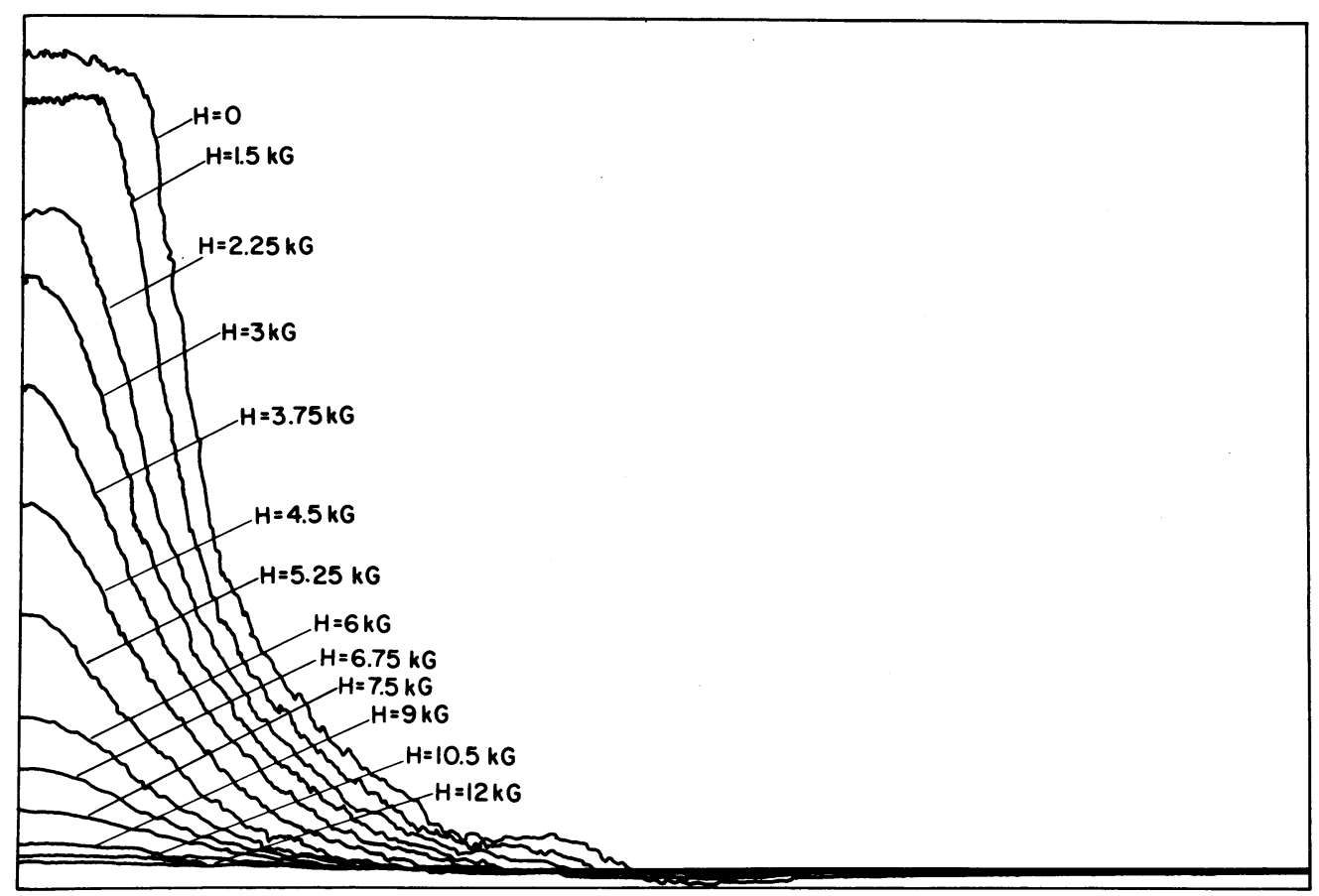

FIGURE 6

$d V / d I-V$ curve for an $\mathrm{Al}-\mathrm{Al}_{2} \mathrm{O}_{3}-\mathrm{Pb}-\mathrm{Pt} \mathrm{junction}$ at $1.1^{\circ} \mathrm{K}$ as a function of a parallel magnetic field (in kilooersteds). The $d V / d I$ scale is $2 \mu \mathrm{V}$ per large division and the voltage scale is $0.2 \mathrm{mV}$ per large division.

$$
d_{\mathrm{Pb}}=1200 \stackrel{\circ}{\AA} ; \quad d_{\mathrm{Pt}}=200 \stackrel{\circ}{\mathrm{A}} \text {. }
$$

\section{Discussion}

\section{(a) Gaps in "normal" metals}

The gaps induced in the three "normal" metals investigated here are listed in Table 1. The coherence distance $\xi$ shown in that table was calculated from the relation:

$$
\xi=\left(\pi \hbar k_{B} \sigma / 40 e^{2} \gamma \Delta\right)^{1 / 2} \text {. }
$$

where $\sigma$ is the low temperature conductivity in absolute units, $\gamma$ the coefficient of normal electronic specific heat in ergs $/{ }^{\circ} \mathrm{K}^{2} \mathrm{~cm}^{3}$ and $2 \Delta$ is the value of the gap generated in the normal metal in $\mathrm{mV}$. Relation (1) was obtained by substituting for $T_{c}$ the B.C.S. relationship $T_{c}=$ $\Delta / 1.76 k_{B}$ in the relation [7]

$$
\xi=\left(\pi \hbar k_{B} \sigma / 6 T_{c} e^{2} \gamma\right)^{1 / 2} \text {. }
$$

It is apparent from relation (1) that the coherence distance $\xi$ has an implicit dependence on 
the normal film thickness via $\Delta$. The experimental values of the conductivities for $\mathrm{Al}, \mathrm{Cd}$ and Pt used in relation (1) were, respectively: $4.7 \times 10^{15}, 5.4 \times 10^{15}$ and $3 \times 10^{16}$ absolute units. The values of $\gamma$ as obtained from the literature [24] were as follows: $\gamma_{\mathrm{Al}}=1.46 \times 10^{3} \mathrm{ergs} /$ ${ }^{\circ} \mathrm{K}^{2} \mathrm{~cm}^{3}, \gamma_{\mathrm{Cd}}=5.46 \times 10^{2} \mathrm{ergs} /{ }^{\circ} \mathrm{K}^{2} \mathrm{~cm}^{3}$ and $\gamma_{\mathrm{Pt}_{\mathrm{t}}}=7.02 \times 10^{3} \mathrm{ergs} /{ }^{\circ} \mathrm{K}^{2} \mathrm{~cm}^{3}$. The experimental

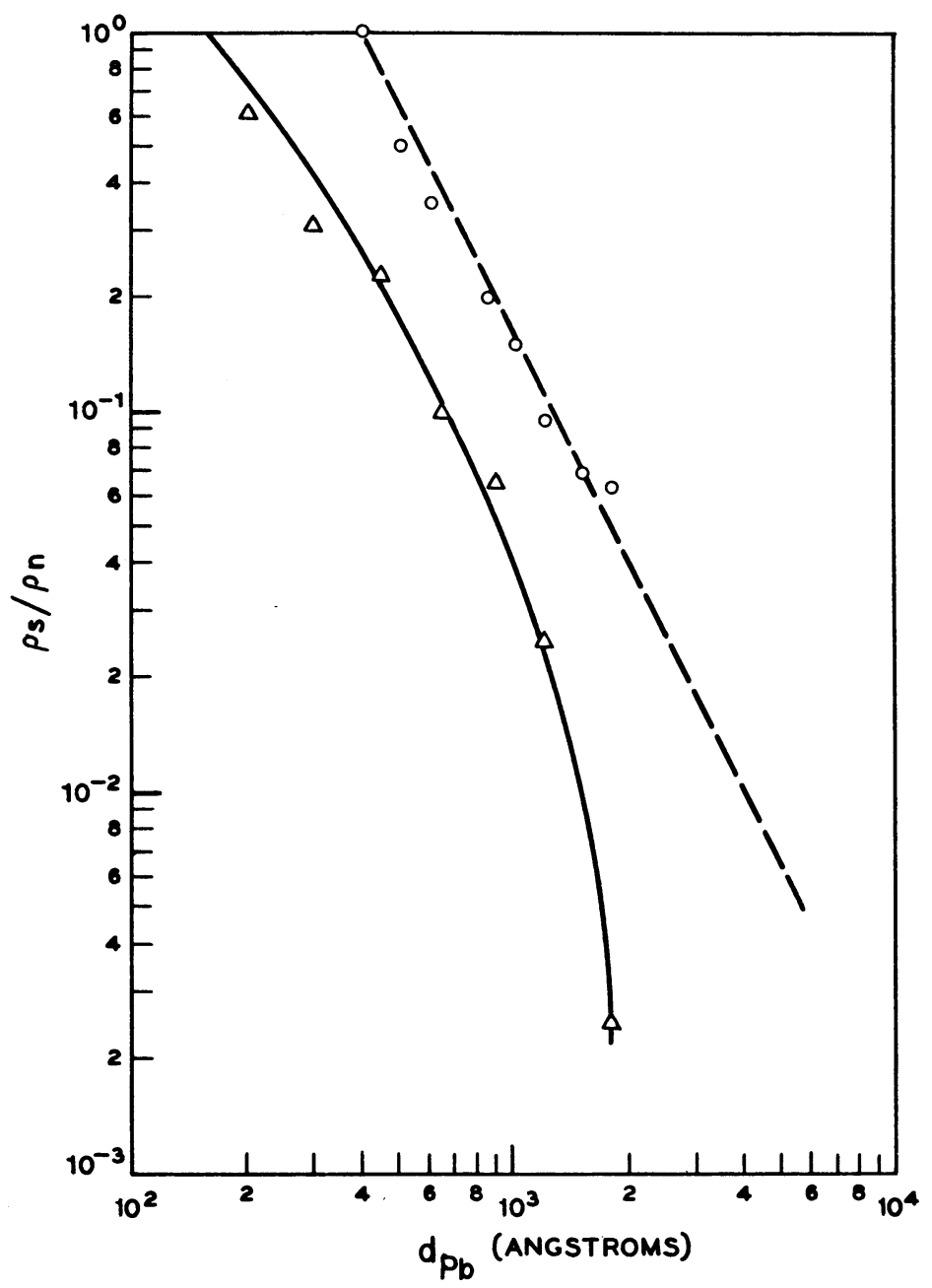

FIGURE 7

Plot of $\log \rho_{s} / \rho_{n}$ as a function of the thickness of the lead film with $d_{N_{i}}=45 \AA$ (dashed curve) and $d_{P_{t}}=200 \AA$ (solid curve).

results shown in Table 1 can be interpreted in the light of two theoretical models $[13,14]$. Saint-James [14] predicts that a gap can only exist in a metal if this metal possesses an attractive electron-electron interaction. Furthermore, if two superconductors $S_{1}$ and $S_{2}$ with respective gaps $\Delta_{1}$ and $\Delta_{2}$ (where $\Delta_{1}<\Delta_{2}$ ) are in contact, his theory asserts that the gap in the energy spectrum will be $\Delta_{1}$ irrespective of the thicknesses of the two superconductors. The results in Table 1 are in clear disagreement with such a theory since the gaps induced in the normal metals are a function of the normal film thickness and gaps approaching that of lead have been measured when the $\mathrm{Al}$, Cd or Pt films were made very thin. The disagreement between this theory and experiment may be due to the fact that the theory was derived under the 
assumption of specular reflexion which is most probably not satisfied experimentally.

McMillan [13] has derived a theory for pure films $(d<\xi)$, where scattering is assumed to take place at the boundary as a result of the mismatch of the density of states of the two

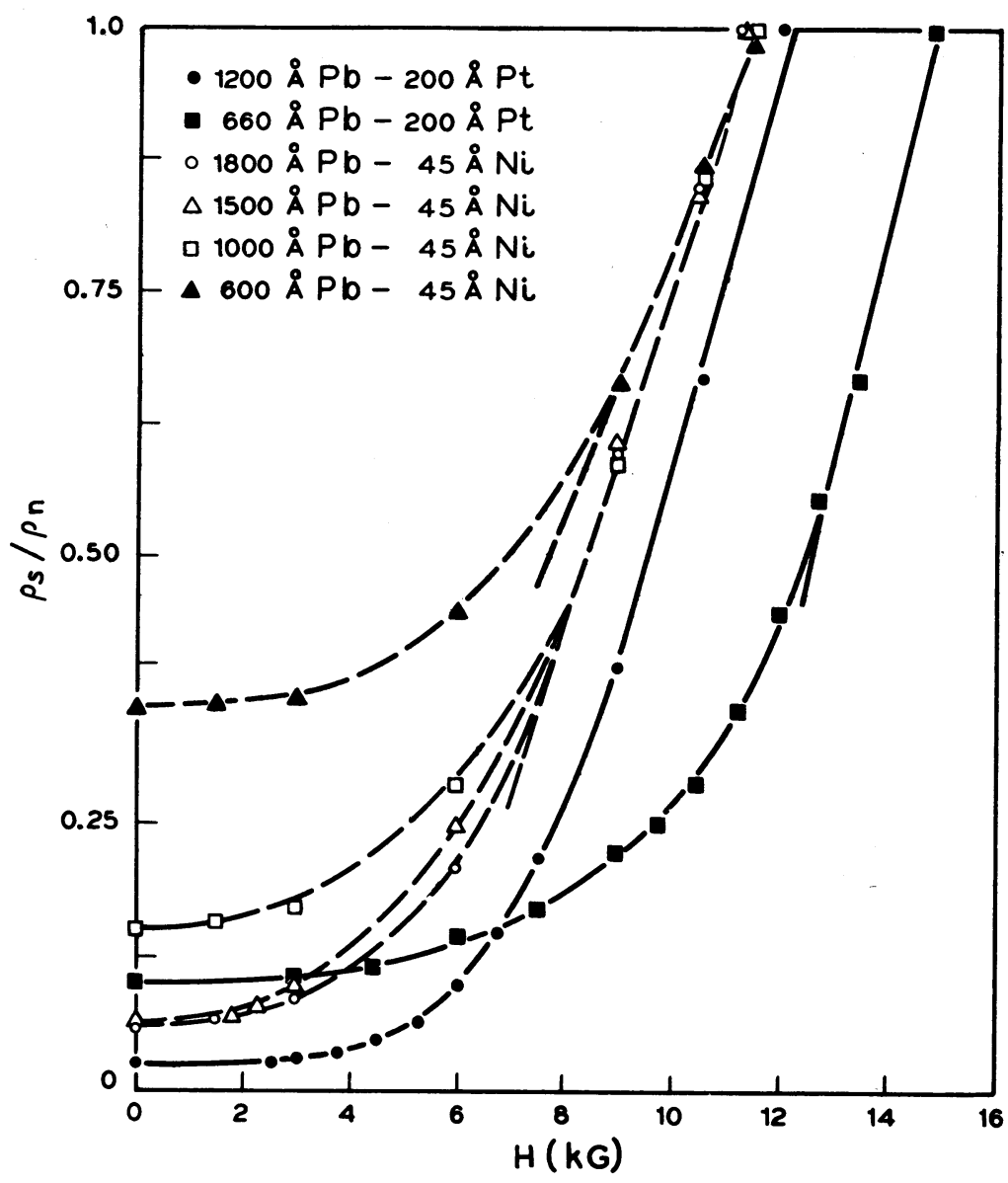

FIGURE 8

$\rho_{s} / \rho_{n}$ as a function of magnetic field (longitudinal field). The solid curves represent the dependence for the $\mathrm{Pb}-\mathrm{Pt}$ sandwiches and the dashed curves the dependence for the $\mathrm{Pb}-\mathrm{Ni}$ sandwiches.

adjoining metals. The requirement of pure films is difficult to satisfy experimentally, as films composing a sandwich must be deposited at low temperature in order to avoid unwanted chemical and metallurgical effects [9]. As a result of his assumption, McMillan's theory is independent of mean free path and leads to the following dependence of induced gap on film thickness

$$
t=\left(\delta-\delta_{0}\right)[(1+\delta) /(1-\delta)]^{1 / 2}
$$

where $\delta=\Delta_{\mathrm{n}} / \Delta_{\mathrm{Pb}}, \quad \delta_{0}=\Delta_{\mathrm{n}}^{0} / \Delta_{\mathrm{Pb}}$ and $t=\hbar V_{\mathrm{F}} p / 2 d_{\mathrm{n}} \Delta_{\mathrm{Pb}} ; \Delta_{\mathrm{n}}$ is the induced gap in the "normal" metal. $\Delta_{n}{ }^{0}$ is the gap present in the "normal" metal in the absence of lead and $p$ is the transition probability for an electron pair to cross the boundary. The quantity $t$ is dimensionless and can 
be written as $d_{0} / d_{n}$ where $d_{0}=\hbar v_{F} p / 2 \Delta_{\mathrm{Pb}} \simeq \xi_{0} p$ is a characteristic length. A plot of the data in terms of the above defined parameters is shown in Fig. 9. In all cases $\Delta_{\mathrm{Pb}}=1.5 \mathrm{mV}$ was used; for Pt $\delta_{0}=0$, for Al $\delta_{0}=0.22$ where the measured value $\Delta_{\mathrm{A} 1}^{\circ}=0.33 \mathrm{mV}$ was used and in the case

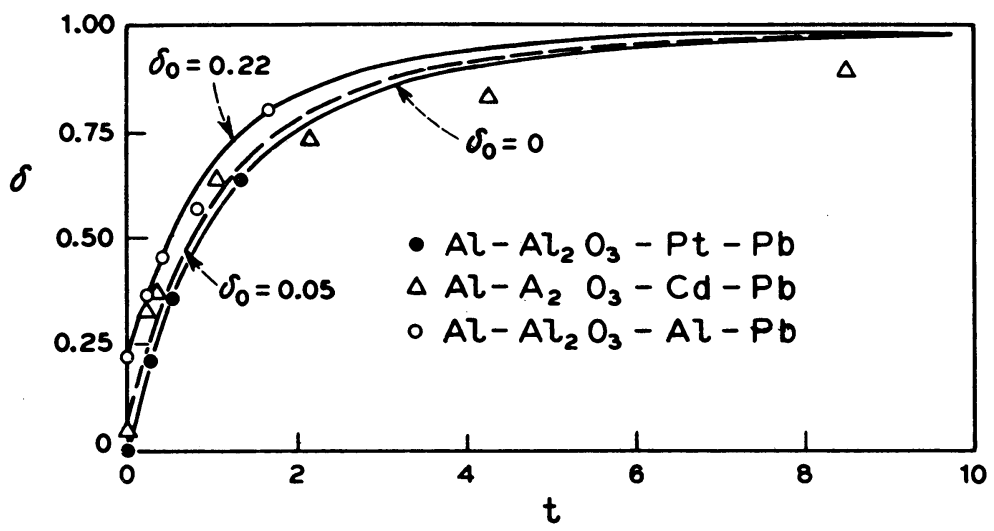

FIGURE 9

Gaps induced in $\mathrm{Al}, \mathrm{Cd}$ and $\mathrm{Pt}$ films by a $900 \AA \mathrm{A}$ lead film as a function of their film thickness $\delta=\Delta_{n} / \Delta_{\mathrm{Pb}}, t=d_{0} / d_{n}$ and $\delta_{0}=\Delta_{n}^{0} / \Delta_{\mathrm{Pb}}$ where $\Delta_{n}$ is the induced gap in the "normal" f1lm, $\Delta_{n}^{0}$ is the gap present in the "normal" metal without the overlying lead and $d_{0}$ is a characteristic length.

of Cd $\delta_{0}=0.05$ was obtained by calculating $\Delta_{\mathrm{Cd}}^{\circ}$ from the measured $T_{c}=0.52^{\circ} \mathrm{K}$ [25] using the usual B.C.S. relation. The value of $d_{0}$ was chosen in each case so that the theoretical curve represents the best average of the data. The values of $d_{0}$ used to $f$ it the data were, respectively, $18 \AA$ for Pt, $58 \AA$ for $A 1$ and $120 \AA$ for Cd. As can be seen from Fig. 9, the agreement between experiment and theory is fairly good. The agreement is best for Pt which as seen from Table 1 best satisfies the pure film assumption $(\xi / d>1)$ under which the theory was derived. The worst agreement is shown by Cd, but even in that case, a correction of 10 per cent at most on the measured value of the gaps would put every point on the curve. Some of the discrepancy between theory and experiment can be attributed to the difficulty in precisely estimating film thickness and the gap value $\Delta_{n}$. The major source of the difficulty, however, must be due to the dirty superconductor nature of most films which will lead to internal scattering, a fact which has not been taken into consideration by the McMillan theory. The characteristic length $d_{0}$ is different for different "normal" metals and one expects that $d_{0}$ will vary mainly with $v_{F}$; the only statement that one can make about $p$ is that it is a number smaller than unity. The experiments agree, therefore, with the McMillan theory to the extent that the experimental values of $d_{0}$ previously quoted are approximately inversely proportional to $\gamma$ (i.e. proportional to $v_{F}$ ). These experiments also agree with the data on $\mathrm{Pb}-\mathrm{Ag}$ sandwiches deposited at room temperature by Rowell [13] who found $d_{0}=200 \AA$ in that case. The density of states of silver is very close to that of cadmium and the difference in $d_{0}$ in these two cases can be attributed to a different value of $p$ resulting from the different preparation method of the sandwiches (room temperature versus liquid nitrogen deposition).

The experiments just described and McMillan's theory raise the possibility that one could use such techniques to predict unknown transition temperatures. The scatter shown in Fig. 9 suggests, however, that such predictions will have to be very qualitative in nature, and not any more accurate than those made by the more conventional proximity effect measurements $[6,7]$. 
It is possible that a theory which would take into account internal scattering would permit more accurate estimates; but the predictions would still be limited by the fact that the induced gap is much more sensitive to variations of such physical properties as film thickness, film resistivity and gap measurements than to the intrinsic gap $\Delta_{n}^{\circ}$.

\section{(b) Gapless superconductivity in lead films}

Although gapless superconductivity has been reported previously in superimposed films of a magnetic metal on a superconductor [15], no systematic study of the effect has yet been reported. An example of such gapless superconductivity is shown for a $\mathrm{Pb}-\mathrm{Ni}$ sandwich in Fig. 4. The degree of gapless superconductivity can be measured by the conductance ratio $\rho_{s} / p_{n}=$ $\left[d V / d I\left(V_{\mathrm{Bias}}=3 \mathrm{mV}\right)\right] /\left[d V / d I\left(V_{\mathrm{Bias}}=0\right)\right]$. As can be seen from Fig. 4 an ambiguity arises in determining $d V / d I$ at zero bias, as it increases very rapidly when the temperature is lowered below $1.4^{\circ} \mathrm{K}$. This effect was at first thought to be caused by nickel as it was not present in a simple $\mathrm{Al}-\mathrm{Al}_{2} \mathrm{O}_{3}-\mathrm{Pb}$ junction. It is striking, however, that this effect always appears below $1.4^{\circ} \mathrm{K}$ (the transition temperature of aluminum) as shown in Fig. 4, while it disappears at $1.1^{\circ} \mathrm{K}$, when a parallel magnetic field of about $2 \mathrm{kG}$ has been applied $(2 \mathrm{kG}$ being approximately the critical field of aluminum at $1.1^{\circ} \mathrm{K}$ ). Furthermore, the lead film used in the experiment described in Fig. 5 was thick enough that some of the electron pairs far from the nickel still display a gap as shown by the peak in $d V / d I$ at $1.2 \mathrm{mV}$. It is noteworthy to point out that this

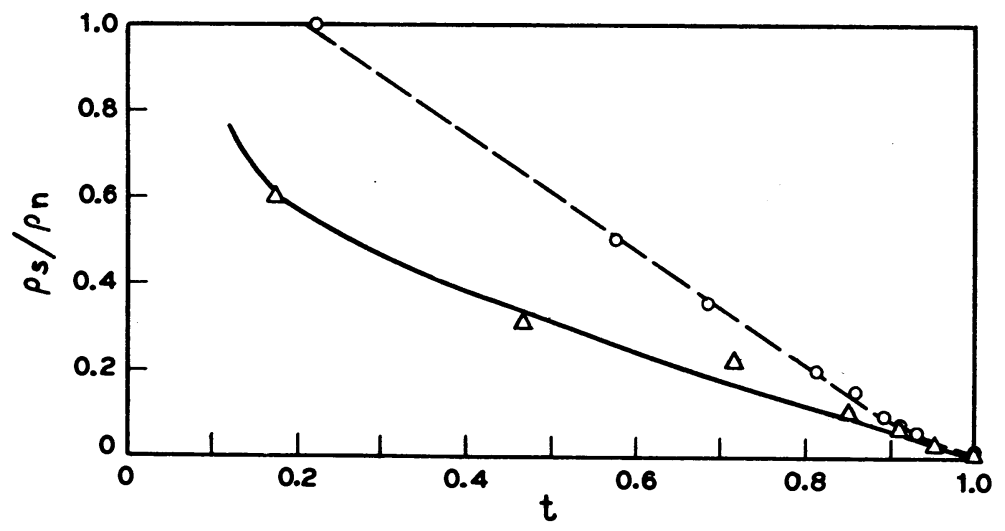

FIGURE 10

$$
\begin{aligned}
& P_{s} / P_{n} \text { as a function of the reduced transition temperature of the sandwich } \\
& \text { with } d_{N_{i}}=45 \AA \text { (dashed curve) and } d_{P_{t}}=200 \AA \text { (solid curve). }
\end{aligned}
$$

peak, which occurs at a voltage between the sum and difference of the lead and aluminum gaps, disappears also at about $2 \mathrm{kG}$. The increase in $d V / d I$ at zero bias below $1.4^{\circ} \mathrm{K}$ is therefore the result of the aluminum gap opening up; and the reason it is not seen in a simple $\mathrm{Al}-\mathrm{Al}_{2} \mathrm{O}_{3}-\mathrm{Pb}$ junction is that there are no states present in the lead gap to reveal it [26]. Consequently, if one does not want the value of $\rho_{s} / \rho_{n}$ to be complicated by the aluminum gap effect, it should be measured slightly above or at $1.4^{\circ} \mathrm{K}$, or at $1.1^{\circ} \mathrm{K}$ with a magnetic field sufficient to destroy the aluminum superconductivity (about $2 \mathrm{kG}$ ). The two values thus obtained are usually very similar, and their average was used to establish the variation of $\rho_{s} / \rho_{n}$ as a function of lead film thickness shown by the dashed line of Fig. 7 . 
Fulde and Maki [16] have predicted that gapless superconductivity would also occur in a superconducting film in contact with a nonmagnetic normal film close to the transition temperature of the superconductor. On the other hand, de Gennes and Mauro [17] have shown that in such normal contacts, the superconductor would always retain an energy gap at all temperatures as long as the normal metal thickness remains finite. In the present experiments, the platinum film is $200 \AA$ thick (about 5 coherence lengths) and, consequently, the gap will be extremely small and as a result of thermal smearing, undetectable experimentally. Thus, since the platinum film can be considered as quasi-infinite from the point of view of the de Gennes-Mauro theory [17], one expects that $\mathrm{Pb}-\mathrm{Pt}$ sandwiches made with such platinum films will be gapless. An example of such gapless superconductivity is shown in Fig. 6. The variation of $p_{s} / p_{n}$ as a function of lead film thickness for Pb-Pt sandwiches is shown as the solid curve of Fig. 7. As one would expect, the effect of nickel is greater than that of a nonmagnetic metal such as platinum. The comparison presented in Fig. 7 contains implicitly yet another effect, namely that all other parameters being the same, nickel depresses the transition temperature of a superconductor more than platinum $[8,9]$. This effect has been taken out in Fig. 10 which shows the variation of $P_{s} / P_{n}$ as a function of the reduced transition temperature of the sandwich for $\mathrm{Pb}-\mathrm{Pt}$ sandwiches (solid curve) and $\mathrm{Pb}-\mathrm{Ni}$ sandwiches (dashed curve). Figure 10 clearly shows that even for sandwiches with the same reduced transition temperature, nickel introduces more states into the lead gap than platinum.

As shown in Fig. 7, the data for $\mathrm{Pb}-\mathrm{Ni}$ sandwiches can be fitted with fair accuracy by the empirical relation:

$$
\rho_{s} / p_{n}=\left(400 / d_{P_{b}}\right)^{2}
$$

with $d_{\mathrm{Pb}} \geqslant 400 \AA$ and $d_{\mathrm{Pb}}$ expressed in angstroms. It has been shown previously [9] that in the case of a proximity effect with a strong magnetic element such as nickel the proximity effect can be described by the single relationship:

$$
\ln T_{c s} / T_{c}=x\left(\pi^{2} \xi_{s}^{2} / 4 d_{\mathrm{Pb}_{b}}^{2}\right)
$$

If one uses the simplifying assumption $x(z) \simeq \ln \left[1+\left(\pi^{2} / 4\right) z\right]$ relation (5) reduces to:

$$
t=1-\pi^{4} \xi_{s s}^{2} / 16 d_{\mathrm{Pb}}^{2}
$$

where $t=T_{c} / T_{c s}$ and $\xi_{s s}$ is the coherence distance of lead measured at $7.2^{\circ} \mathrm{K}(140 \AA)$. Elimination of $d_{\mathrm{Pb}}$ between relations (4) and (6) leads to the linear equation:

$$
p_{s} / p_{n}=1.30(1-t)
$$

which again fits fairly well with the $\mathrm{Pb}-\mathrm{NI}$ data shown in Fig. 10.

Gapless superconductivity can also occur in thin "dirty" films under high magnetic fields [19-21]. Experiments to demonstrate that are shown in Fig. 8. In the present case, the samples are gapless even in zero magnetic field as a result of the proximity effect previously discussed. The magnetic field increases the degree of gapless superconductivity, and one can observe in Fig. 8 that $\rho_{s} / \rho_{n}$ is a linear function of magnetic field close to the upper critical field. A similar experimental observation has been reported on bulk type II superconductors $[22,23]$. It has been pointed out [27] that the study of gapless superconductivity in thin films under uniform magnetic fields may be difficult as the results are sensitive to possible modulations of the film thickness. Such difficulty is obviated if one uses sandwiches instead of thin 
films as in that case the film thickness can be larger than the penetration depth. Although the upper critical fields of the various sandwiches shown in Fig. 8 are different, the slopes of $\rho_{s} / P_{n}$ versus magnetic field curves are nearly the same for all $\mathrm{Pb}-\mathrm{Pt}$ and $\mathrm{Pb}-\mathrm{Ni}$ sandwiches. Such behavior was expected from the fact that the effect of the field is only through the average of $|\Delta|^{2}$ at the surface near the junction [27]. As the transition near the upper critical field is of second order, one expects $\left\langle|\Delta|^{2}\right\rangle$ to be linear in the magnetic field in that region as derived by Maki [28]. As shown by the similar curves obtained on the $1800 \AA \mathrm{Pb}-200 \AA \mathrm{Pt}$ sandwich on the one hand and the $1800 \AA \mathrm{Pb}-45 \AA \mathrm{Ni}$ sandwich on the otner, the effect of the magnetic field on gapless superconductivity is quite independent of the nature of the sample used.

\section{Acknowledgments}

It is with great pleasure that I thank W.L. McMillan for making available to me his theory prior to publication and for several helpful suggestions. I would also like to thank J.M. Rowell, S. Shapiro, D.E. Thomas and H.C. Theuerer for their suggestions on tunneling techniques. Finally, I would like to express my gratitude to D.D. Bacon who patiently produced all the tunnel junctions used in this study and to W.H. Haemmerle for his technical assistance in obtaining the data.

\section{References}

1. P. HILSCH, Z. Phys. 167, 511 (1962).

2. P. HILSCH and R. HILSCH, Z. Phys, 180, 10 (1964).

3. P.G. de GENNES and E. GUYON, Phys. Letters 3, $168(196 \dot{3})$.

4. P.G. de GENNES, Rev. Mod. Phys. 36, 225 (1964).

5. N.R. WERTHAMER, Phys. Rev. 132, 2440 (1963).

6. J.J. HAUSER, H.C. THEUERER and N.R. TERThAMER, Phys. Rev. 136, A637 (1964).

7. J.J. HAUSER and H.C. THEUERER, Phys. Letters 14, 270 (1965).

8. J.J. HAUSER, H.C. THEUERER and N.R. WERThAMER, Phys. Letters 18, 222 (1965).

9. J.J. HAUSER, H.C. THEUERER and N.R. WERTHAMER, Phys. Rev. 142, 118 (1966).

10. G. BERGMANN, Z. Phys. 187, 395 (1965).

11. H. MEISSNER, Phys. Rev. 117, 672 (1960).

12. P.H. SMITH, S. SHAPIRO, J.L. MILES and J. NICOL, Phys. Rev. Letters 6, 686 (1961).

13. W.L. MCMILLAN and J.M. ROWELL. Experiments and theory will be published in this journal at a future date.

14. D. SAINT-JAMES, J. de Phys. 25, 899 (1964). 
15. M.A. WOOLF and F. REIF, Phys. Rev. 137, A557 (1965).

16. P. FULDE and K. MAKI, Phys. Rev. Letters 15, 675 (1965).

17. P.G. de GENNES and S. MAURO, Solid State Communications 3, 381 (1965).

18. I.S. KHUKHAREVA, Zh. Eksp. Teor. Fiz. 43, 1173 (1962); Sov. Phys.-JETP 16, 828 (1963).

19. K. MAKI, Prog. Theor. Phys. 31, 731 (1964).

20. P.G. de GENNES and M. TINKHAM, Physics 1, 107 (1964).

21. P. FULDE, Phys. Rev. 137, A783 (1965).

22. W. TOMASCH, Phys. Letters 9, 104 (1964).

23. E. GUYON, A. MARTINET, J. MATRICON and P. PINCUS, Phys. Rev. 138, A746 (1965).

24. C. KITTEL, Introduction to Solid State Physics, p. 259. John Wiley (1957).

25. I am indebted to G.W. HULL, Jr. and T.H. GEBALLE for this measurement.

26. This explanation was suggested to me by P.W. ANDERSON.

27. Sussex Symposium on Quantum Fluids, University of Sussex, September 1965; Orsay Group paper to be published.

28. K. MAKI, Physics 1, 21 (1964). 